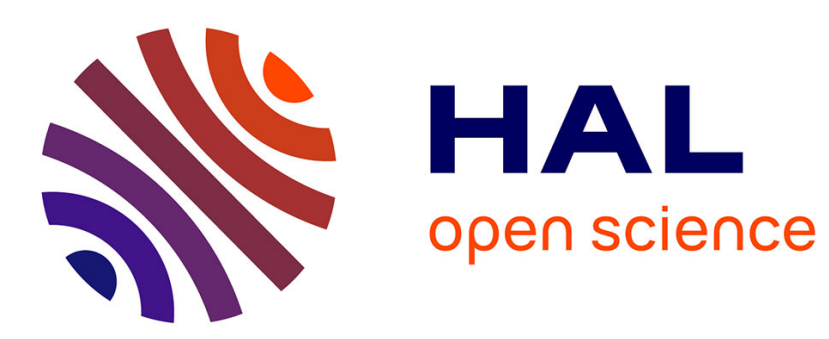

\title{
De la méthode critique au doute compulsif
}

\author{
Jacques Barou
}

\section{To cite this version:}

Jacques Barou. De la méthode critique au doute compulsif. L'école des parents, 2021. hal-03537294

\section{HAL Id: hal-03537294 \\ https://hal.science/hal-03537294}

Submitted on 20 Jan 2022

HAL is a multi-disciplinary open access archive for the deposit and dissemination of scientific research documents, whether they are published or not. The documents may come from teaching and research institutions in France or abroad, or from public or private research centers.
L'archive ouverte pluridisciplinaire HAL, est destinée au dépôt et à la diffusion de documents scientifiques de niveau recherche, publiés ou non, émanant des établissements d'enseignement et de recherche français ou étrangers, des laboratoires publics ou privés. 


\section{De la méthode critique au doute compulsif}

«Ce n'est pas le doute, c'est la certitude qui rend fou. », ${ }^{1}$ nous dit Nietzsche, réfléchissant aux conditions nécessaires à la démarche philosophique. L'affirmation es-elle valable pour les adolescents vivant à l'ère d'internet ?

Cet aphorisme de Nietzsche, extrait du plus autobiographique de ses ouvrages, Ecce homo, incite à remettre en question les certitudes héritées pour tracer soi-même son propre chemin vers la vérité. Dans cette démarche, le doute est un allié utile. Encore faut-il en user avec pertinence, pour qu'il ne mène pas à une paralysie de la pensée. L'esprit critique se construit dans cet intervalle entre des dogmes imposés d'en haut et une remise en cause qui doit s'appuyer sur un minimum de vérités acquises afin de pouvoir procéder par paliers. Si la critique de la raison est, comme l'a écrit $\mathrm{Kant}^{2}$, «l'examen des pouvoirs et des limites de la raison », l'esprit critique consiste à examiner ce qui, dans une affirmation énoncée, résiste au doute et ce qui n'y résiste pas, plutôt que de remettre en cause brutalement et globalement cette affirmation. Cette démarche est présente dès l'aube de la philosophie occidentale, même si on ne l'appelle pas encore critique.

Dans sa fameuse allégorie de la caverne ${ }^{3}$, Platon met en scène des prisonniers enchaînés au fond d'une grotte : leur tête est immobilisée de sorte qu'ils ne peuvent regarder que vers le fond de la caverne où, en raison d'un feu allumé derrière eux, ils ne voient que leurs ombres, qu'ils prennent pour des personnages réels. Ils sont donc doublement prisonniers, enchaînés sur le plan physique et intellectuellement soumis à une certitude illusoire. Imaginons qu'on délie l'un d'entre eux et qu'on l'amène au dehors pour lui montrer des êtres réels éclairés par la lumière du jour: il sera d'abord ébloui par l'éclat du soleil et en souffrira, comme il souffrira de constater que ce qu'il prenait pour la réalité n'était qu'une illusion et il sera tenté de retourner dans la caverne retrouver ses ombres familières. S'il essaye de convaincre ses compagnons que ce qu'ils croient vrai n'est que l'apparence tronquée de la vérité et que leurs certitudes sont erronées, il provoquera leurs moqueries. S'il insiste, peut-être préfèreront-ils le tuer plutôt que de remettre en cause leurs croyances.

Cette allégorie vise avant tout à illustrer la théorie de la connaissance de Platon : il faut se défier de ses sens et tenter de connaître l'essence des choses par la raison. La connaissance avance au rythme de la dialectique, par étapes successives : le prisonnier libéré découvre d'abord les êtres puis le soleil qui les éclaire et les fait vivre. On peut voir là l'amorce d'une démarche critique. Pour le lecteur d'aujourd'hui, ce texte est une belle illustration du poids des fausses certitudes, du conformisme des masses et de l'importance de la liberté pour accompagner l'accès à la vérité. L'aveuglement est aliénant et celui qui a découvert le «vrai » ne peut plus revenir en arrière : "Ne préférera-t-il pas mille fois n'être qu'un valet de charrue, au service d'un pauvre laboureur, et de souffrir tout au monde plutôt que de revenir à ses anciennes illusions et vivre comme il vivait?»

\footnotetext{
${ }^{1}$ Friedrich Nietzsche, Ecce homo. Comment on devient ce que l'on est, in Euvres (Gallimard, coll. « La Pléiade », 2000).

${ }^{2}$ Emmanuel Kant, Critique de la raison pure (Flammarion, 2006).

${ }^{3}$ Platon, La République, livre VII (Flammarion, 2016).
} 


\section{Totalitarisme et esprit critique}

Les prisonniers de Platon, aussi étranges soient-ils, sont comparables aux hommes qui se pensent libres alors qu'ils se conforment à des vérités héritées sans les interroger. Au temps de Platon, les citoyens d'Athènes étaient la cible des sophistes dans un but électoral. Ces habiles orateurs, souvent au service du pouvoir politique, tenaient des discours qui leur permettaient de substituer la séduction verbale à l'esprit critique. Mais l'organisation démocratique de la cité favorisait la confrontation des opinions et l'émergence, au-dessus du lot de la pensée philosophique, argumentative et rationnelle, pour définir une «opinion vraie », aboutissement d'une démarche critique.

Est-ce à dire qu'exercer l'esprit critique n'est possible que dans une démocratie, ou qu'il suffit de vivre en démocratie pour en être capable? L'exercice de la critique se confronte inévitablement à des enjeux de pouvoir, que ce soit dans le domaine public ou dans le domaine privé. Au sein de la cellule familiale, les principes transmis se veulent souvent incontestables et ne peuvent être interrogées par personne, surtout pas par les jeunes générations, censées se soumettre aux anciens. Cela peut expliquer l'hostilité que rencontre un certain type d'enseignement de la part des familles dans des sociétés plutôt conservatrices, malgré l'intérêt qu'elles accordent à l'éducation. Dans les années 1970, le Niger, État africain récemment indépendant, a tenté de développer un enseignement par radio destiné aux enfants des peuples nomades du nord du pays ${ }^{4}$. Le succès a été immédiat mais il a fallu interrompre l'expérience à la demande des grands chefs touareg, qui recevaient des plaintes de parents dont les enfants, depuis qu'ils écoutaient l'école à la radio, ne cessaient de leur poser des questions. Cette attitude était perçue non comme un éveil de la pensée réflexive, mais comme une remise en cause intolérable du pouvoir des adultes, lequel reposait sur des principes qu'eux-mêmes ne s'autorisaient pas à interroger. La perpétuation de l'ordre établi, qu'il soit familial, social ou politique, est un objectif qui écarte toute remise en question favorisant le développement de l'esprit critique. Sous les régimes totalitaires, la moindre contestation est perçue par le pouvoir en place comme une forme de dissidence qui sape les fondements de l'ordre établi. De ce fait, l'État instaure un contrôle étroit sur les publications à contenu réflexif et surveille les relations que les citoyens peuvent avoir avec le monde extérieur. Cela ne concerne pas seulement les intellectuels qui exportent leurs travaux critiques dans les pays démocratiques et font connaître ainsi le véritable caractère des régimes dictatoriaux mais aussi la masse des citoyens, que le pouvoir s'efforce d'empêcher d'avoir accès aux médias étrangers, par crainte d'une possible contagion démocratique. À l'époque de la guerre froide, dans les pays socialistes, l'accès à des médias comme Radio Free Europe ou The Voice of America était particulièrement surveillé. Comme les prisonniers de la caverne, les citoyens étaient, autant que possible, maintenus dans l'ignorance du monde extérieur. La découverte d'une autre réalité risquait de les amener à interroger celle qu'ils vivaient au quotidien et à critiquer leur gouvernement. Beaucoup de théories ont été proposées pour expliquer la chute des régimes socialistes d'Europe de l'Est. On pourra au moins retenir l'idée que, dans un espace largement ouvert à la communication, il était devenu impossible de préserver une partie de l'Europe de toute contamination par l'esprit critique. Ceux qui étaient au pouvoir se

\footnotetext{
${ }^{4}$ J. Barou, « L'émigration dans un village du Niger », Cahiers d'études africaines, nº 63-64, 1976.
} 
sont eux-mêmes mis à douter du bien-fondé de leur système et de sa capacité à se réformer. Ils n'ont plus voulu faire l'effort de le maintenir en place et encore moins par la censure et la répression.

\section{Le doute compulsif}

L'effondrement de ces régimes s'est produit quelques années avant le développement d'Internet et des réseaux sociaux. Ce nouveau type de média aurait-il pu instiller le doute dans l'esprit de leurs citoyens de manière aussi efficace qu'ont pu le faire les modes de communication classiques presse, radio, télévision, téléphone? Le printemps arabe en 2010 s'est beaucoup appuyé sur les réseaux sociaux pour se propager. Mais ceux-ci ont surtout permis de mobiliser rapidement les opposants aux dictatures en place sans changer en profondeur le regard des masses sur le régime ${ }^{5}$. Les résultats de ce mouvement n'ont finalement abouti qu'au chaos ou au retour des dictatures, sauf peut-être en Tunisie. La volatilité des liens créés sur le net n'est-elle pas préjudiciable à l'action en profondeur que requiert le développement de l'esprit critique ?

À voir certaines théories qui prolifèrent aujourd'hui sur la Toile, on peut se demander au contraire si Internet n'a pas tendance, à l'inverse, à affaiblir l'esprit critique. Les diverses théories du complot qui connaissent le succès auprès des internautes font en effet écran au réel. Leur principe est de remettre systématiquement en cause toute version officielle, qu'il s'agisse d'un événement comme les attentats du 11 septembre 2001 ou de données scientifiques, comme l'ont fait plus récemment les «platistes », qui affirment que la terre est plate et font circuler quantité de vidéos en ce sens sur YouTube ${ }^{6}$. L'incitation à douter ne relève pas là de la démarche critique mais de la volonté de discréditer les positions officielles, présentées comme issues d'un groupe de manipulateurs exerçant un pouvoir occulte sur les masses : satanistes, pédophiles, sionistes, etc... On retrouve là une partie des racines de l'antisémitisme et, plus généralement, du complotisme qui ont nourri certaines idéologies totalitaires du siècle dernier. Même si les internautes sont loin de tous adhérer à de telles opinions, il n'en reste pas moins que le doute systématique peut aussi avoir des incidences déstabilisantes, notamment sur les adolescents, très présents sur les réseaux sociaux et qui vivent déjà une étape du développement de leur personnalité marquée par l'incertitude. Entre la quiétude de l'enfance et la stabilité que confère l'arrivée à l'âge adulte, les jeunes ont besoin de se rattacher à des repères solides. En les incitants à douter de tout, on achève de les désorienter. Seul l'exercice de l'esprit critique par l'examen objectif et la comparaison des thèses proposées, peut les protéger de la contagion du doute compulsif qui risque qui les conduit au nihilisme. Le rôle des parents, des enseignants et tous les éducateurs en position de les aider à construire leur esprit critique par le dialogue et la réflexion est plus que jamais fondamental.

Jacques Barou CNRS UGA

\footnotetext{
${ }^{5}$ D.M Faris, La révolte en réseau : le printemps arabe et les média sociaux, Politique étrangère, printemps 2012, pp 99/109.

${ }^{6}$ Rudy Reichstadt, L’Opium des imbéciles (Grasset, 2019).
} 
